Thorax (1975), 30, 316.

\title{
Idiopathic progressive pulmonary fibrosis
}

\author{
DE W I D VIES, J.S. CR OW THER, \\ and A N D REW M a F ARLANE
}

Ransom Hospital, Mansfield

\begin{abstract}
Davies, D., Crowther, J. S., and MacFarlane, A. (1975). Thorax, 30, 316-325. Idiopathic progressive pulmonary fibrosis. Five patients with progressive fibrotic lung disease are described. The dominant symptom was slowly increasing dyspnoea, and cough and sputum were not prominent. Marked weight loss was also a feature. There was severe restrictive impairment of ventilation with normal arterial gas tensions. The changes were confined to the upper parts of the lung in some but others had more generalized disease. The duration has varied so far from two to 17 years.

The lung changes are considered to be due to dense progressive fibrosis. Necropsy in two confirmed this. Histologically there was monotonous fibrosis with lymphoid collections and secondary bronchiectasis, a picture similar to that found in association with ankylosing spondylitis. None of these patients had joint disease.

Tuberculosis was excluded as a cause by exhaustive bacteriological tests and the failure of chemotherapy to stop deterioration. All other recognized types of infective and non-infective progressive lung fibrosis were also excluded, and this is not considered to be a variant of cryptogenic fibrosing alveolitis. Though these patients have many features in common they do not necessarily have the same pathogenesis. They are presented as an encouragement to further study.
\end{abstract}

Patients with lung fibrosis, especially in the upper lobes, are readily assumed to have tuberculosis. If tubercle bacilli are not found the changes are often attributed to silent tuberculosis which has healed spontaneously. Such an explanation becomes untenable if the disease progresses without evidence of active tuberculosis or other infection. Some other explanations have gradually emerged.

In an investigation of 61 patients with fibrosis and cavitation notified as cases of tuberculosis without tubercle bacilli ever being demonstrated, 26 were considered to be true cases of tuberculosis (Davies, 1970). Bacilli had not been found because of difficulties in obtaining sputum or because the disease appeared to be healed when discovered. Four patients with ankylosing spondylitis had progressive disease which was clearly not due to tuberculosis. It is now established that some patients with spondylitis develop progressive upper lobe fibrosis as an extra-articular manifestation of the disease (Davies, 1972; Crompton, Cameron, and Langlands, 1974).

In the above survey (Davies, 1970) there remained eight patients with progressive disease and no discoverable cause. Tuberculosis was excluded by adequate bacteriological studies, and the on associated condition was psoriasis in one casê Such a disease is not described in textbooks and the only discovered reference consists of two case reports (Clinico-Pathological Conference, 196 1966). This paper describes five further cases.

CASE 1 R.B., born in 1903, had been a coal miner for 27 years until 1947. He was then labourer until 1966 when he was referred with five-year history of mild cough, sputum, an dyspnoea. He smoked 20 cigarettes per day an had mild psoriasis. Apart from a few wheezes there was no other abnormality on examination A chest radiograph taken in 1962 showed a little fibrosis in the right upper lobe, some hazy opacity at the right apex, and slight pleural thickening at the left base. There was no evidence of pneue moconiosis. The 1966 film (Fig. 1) showed exten sion of shadowing throughout the right upper lob $\bar{\theta}$ and the development of some irregular nodulatio $\vec{P}$ in the left upper zone.

It was decided to treat him as a case of indolenf tuberculosis though the failure to find tubercle bacilli suggested that he might have progressive 


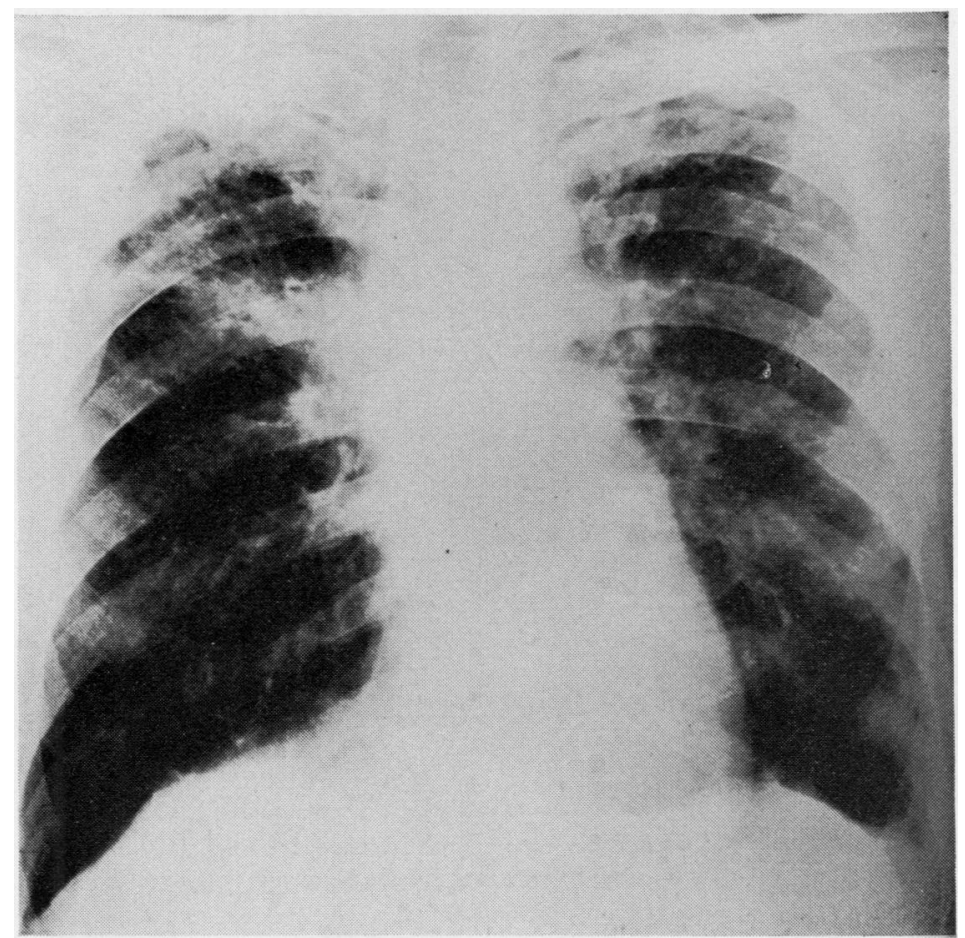

FIG. 1. Case 1. Chest radiograph in 1966 showing shadowing throughout right upper lobe and scattered opacities in the left upper zone.

fibrosis of unknown cause. He was given streptomycin, PAS, and isoniazid for two months, and PAS and isoniazid for 16 months without improvement. Dyspnoea gradually increased and in 1970 the radiograph showed contraction of the right upper lobe with extension of the irregular shadowing on the left to involve the upper half of the lung.

By the end of 1972 he was short of breath on slight exertion. Cough was fairly troublesome and the sputum mainly mucoid. He had lost $10 \mathrm{~kg}$ in weight. There was no clubbing of the fingers. Bronchial breathing and a few crackles were present over both upper zones. The radiograph (Fig. 2) showed the development of some irregular fine opacities in the remainder of the right lung with obliteration of the costophrenic angle and extension of the irregular shadows on the left to involve most of the lung.

He had kept pigeons for a year at the age of 17 but there was no other avian contact. He had not taken any drugs regularly.

Early investigations (1966) Haemoglobin and white blood count were normal; ESR $13 \mathrm{~mm}$ in
$1 \mathrm{hr}$ (Westergren); Rose Waaler and latex negative; Aspergillus fumigatus precipitin and skin prick tests negative; tuberculin skin test strongly positive. Many sputum tests for tubercle bacilli were negative.

Later investigations (1972) Haemoglobin, white blood count, and platelets normal; ESR 27 and $43 \mathrm{~mm}$ in 1 hour; serum proteins $71 \mathrm{~g} / 1$ with an increase in alpha 2 and gamma globulins; alpha 1 antitrypsin normal; Rose Waaler tests positive $1: 16$; latex, antinuclear, mitochondrial and parietal cell antibody tests negative; precipitin tests with pigeon, budgerigar, and hen extracts negative; Kveim test negative; liver function tests, serum urea, and electrolytes normal; electrocardiogram normal; arterial $\mathrm{pH}$ and $\mathrm{PCO}_{2}$ normal, $P_{\mathrm{O}_{2}} 9.44 \mathrm{kPa}(71 \mathrm{mmHg})$; transfer factor (carbon

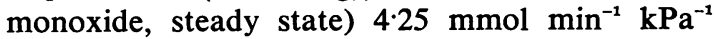
$\left(12.7 \mathrm{ml} \mathrm{min}^{-1}\right.$ torr $\left.^{-1}\right)$ at rest and $7.70 \mathrm{mmol}$ $\min ^{-1} \mathrm{kPa}^{-1}$ (23 $\mathrm{ml} \mathrm{\operatorname {min } ^ { - 1 } \text { torr }}{ }^{-1}$ ) on exercise; sputum tests repeatedly negative for tubercle bacilli; radiographs of pelvis and spine, no evidence of ankylosing spondylitis. 


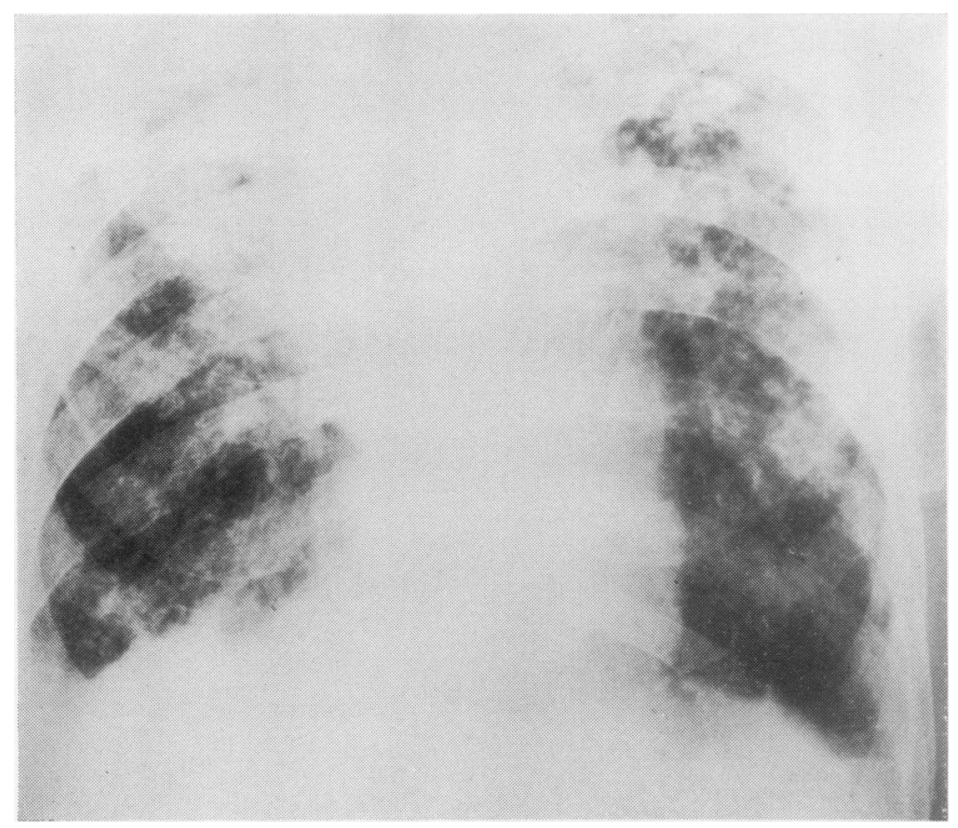

FIG. 2. Case 1. Radiograph in 1972 showing extension of shadowing in the upper zones, the development of fine nodulation in the remainder of the lungs, and obliteration of the costophrenic angles.

Ventilatory tests in 1966 showed fairly severe airways obstruction probably due to chronic bronchitis (VC 1.851 , FEV 1.11 , PEFR $2501 / \mathrm{min}$ ). As the fibrosis progressed the vital capacity fell without any further decrease in $\mathrm{FEV}_{1}$ and peak expiratory flow rate. The 1972 readings were 1.05 , 1.05 , and $2401 / \mathrm{min}$ respectively.

Early in 1973 he was given prednisolone for seven weeks, starting with $30 \mathrm{mg}$ daily. During this period he developed a shallow left apical pneumothorax which absorbed spontaneously. There was no improvement and he died in March 1973.

Necropsy There were dense adhesions in the upper halves of both pleural sacs. The lungs (right $825 \mathrm{~g}$, left $1170 \mathrm{~g}$ ) were distended with formalin and whole lung sections were prepared. Right lung: there was considerable shrinkage of the upper lobe which was almost completely replaced by dense greyish-black fibrous tissue. The bronchi were dilated. Fibrous tissue extended across the fissures into the middle lobe and the apex of the lower lobe (Fig. 3). In the lower lobe there was subpleural fibrosis and honeycombing extending inwards for $2-3 \mathrm{~cm}$ and best seen in the base and posterior margin. Left lung: dense greyish-black fibrous tissue replaced a large part of the upper lobe and extended into the apex of the lower lobe. There was bronchiectasis through out this area. Honeycombing was present subo pleurally in the anterior part of the upper lobe lingula, and base of the lower lobe.

Microscopically the fibrous tissue was compose of dense collagen completely obliterating the framework, apart from occasional small distorte empty air spaces. Foci of lymphocytes were pros minent (Fig. 4). The dilated bronchi containe $\bar{\AA}$ varying amounts of mucopus and there was severe endarteritis obliterans. Around the margins the fibrosis often had an interstitial pattern. In the lower lobes subpleurally there was honeycombin and an overgrowth of muscle, a picture resembling fibrosing alveolitis (Fig. 5).

The heart weighed $300 \mathrm{~g}$ and showed slightes right ventricular hypertrophy. There was no sigg nificant abnormality in the other organs.

CASE 2 E.E., born in 1931, had worked for several years in a dress factory. She had neve smoked. In 1962 she gave one year's history d: slight breathlessness on exertion, cough, scantf mucoid sputum, and a weight loss of $3 \mathrm{~kg}$. Ex amination revealed no abnormality and the radio graph showed a little infiltration in the first righg 


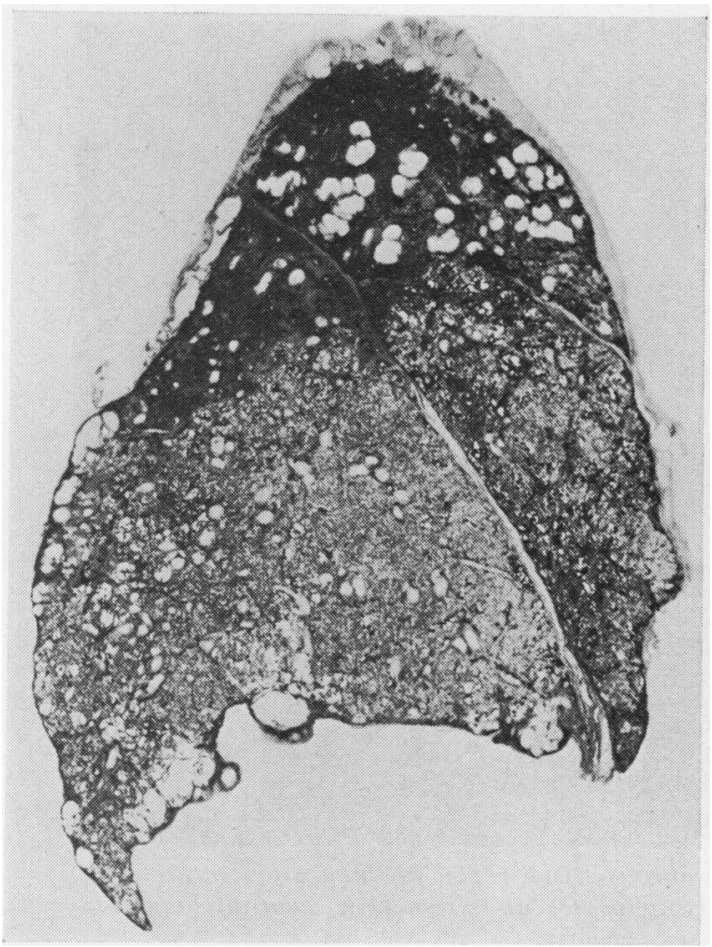

FIG. 3. Case 1. Right lung: Gough-Wentworth whole-lung section. Dense pleural thickening over upper part. Replacement of upper lobe, apex of lower lobe, and part of middle lobe by dense fibrous tissue containing dilated bronchi. Subpleural fibrosis and honeycombing in posterior and basal regions of the lower lobe.

interspace. There was little change until 1966 when she became tired and lost more weight. The radiograph (Fig. 6) showed more shadowing in the periphery of the right upper lobe and fresh shadowing in the left upper/mid-zone. Twelve sputum specimens failed to reveal tubercle bacilli but as she was pregnant she was treated with streptomycin, PAS, and isoniazid for three months. She gave birth to a full-term normal infant and PAS and isoniazid were continued for two years, during which time the fibrosis and breathlessness increased steadily.

By 1973 she was very weak and short of breath on slight exertion. Her weight had dropped from $68 \mathrm{~kg}$ in 1962 to $34 \mathrm{~kg}$. She had a moderate cough and very little sputum and had never wheezed. There was no finger clubbing. Bronchial breathing was present over the upper zone. The radiograph (Fig. 7) showed extensive fibrosis and some honeycombing in the upper halves of the lungs with compensatory emphysema at the bases. She was given $10 \mathrm{mg}$ of prednisolone daily and began to feel stronger and able to get about better. She lost her cough and regained $5 \mathrm{~kg}$ in weight. After a year there was no change in her ventilatory tests or radiographs and she continued on $7.5 \mathrm{mg}$ of prednisolone per day.

A budgerigar had been kept at her home when she was a child and for four weeks in 1961.

Investigation Haemoglobin and white blood count were repeatedly normal but an eosinophilia of $20 \%$ and $21 \%$ was reported on two occasions out of eleven; ESR $18 \mathrm{~mm}$ in $1 \mathrm{hr}$, rising to $66 \mathrm{~mm}$ in 1972; serum albumin $37 \mathrm{~g} / 1$, globulin $40 \mathrm{~g} / 1$ with a slight increase in beta and gamma globulins; IgA $4.4 \mathrm{~g} / 1$ (slightly raised), IgG $34 \mathrm{~g} / 1$ (raised), and IgM 1.5 g/l; alpha 1 antitrypsin level normal; Rose Waaler and latex tests negative, test for thyroid, parietal cell, mitochondrial, and smooth muscle antibodies negative, antinuclear factor negative; precipitin tests with avian and $A$. fumigatus extracts negative; prick tests with $A$. fumigatus, hen, pigeon, and budgerigar extracts negative; tuberculin skin test moderately positive; 40 sputum tests for tubercle bacilli negative, no aspergilli isolated, and no eosinophils seen; no radiographic evidence of ankylosing spondylitis.

In 1969 the arterial $\mathrm{pH}, \mathrm{PCO}_{2}$, and $\mathrm{PO}_{2}$ were normal and the carbon monoxide diffusing capacity (steady state) was $5.02 \mathrm{mmol} \mathrm{min}{ }^{-1} \mathrm{kPa}^{-1}$ $\left(15 \mathrm{ml} \mathrm{min}^{-1}\right.$ torr $\left.^{-1}\right)$ at rest and $8.37 \mathrm{mmol} \mathrm{min}^{-1}$ $\mathrm{kPa}^{-1}\left(25 \mathrm{ml} \mathrm{min}^{-1}\right.$ torr $^{-1}$ ) after exercise (moderately reduced). Between 1969 and 1973 her vital capacity fell from 0.95 litres to 0.4 litres and the $\mathrm{FEV}_{1}$ from 0.6 litres to 0.35 litres. The electrocardiogram showed right atrial hypertrophy.

CASE 3 C.B., a housewife born in 1912, was seen in 1964 complaining of slight cough and sputum and a little dyspnoea for four years. She was thin and the only clinical abnormality was harsh breath sounds over the upper zones. Radiographs showed the hila to be pulled upwards, patchy dense opacities at both apices, and some peripheral consolidation in the axillary area of the right upper lobe. There was also a little patchy shadowing in the right costophrenic angle. There was little change in her symptoms except for a small haemoptysis in 1967. By that time there was patchy consolidation and fibrosis affecting most of the right upper lobe. On the left there had been extension of the shadowing in the upper zone, in the periphery of the mid zone, and in the costophrenic angle. 


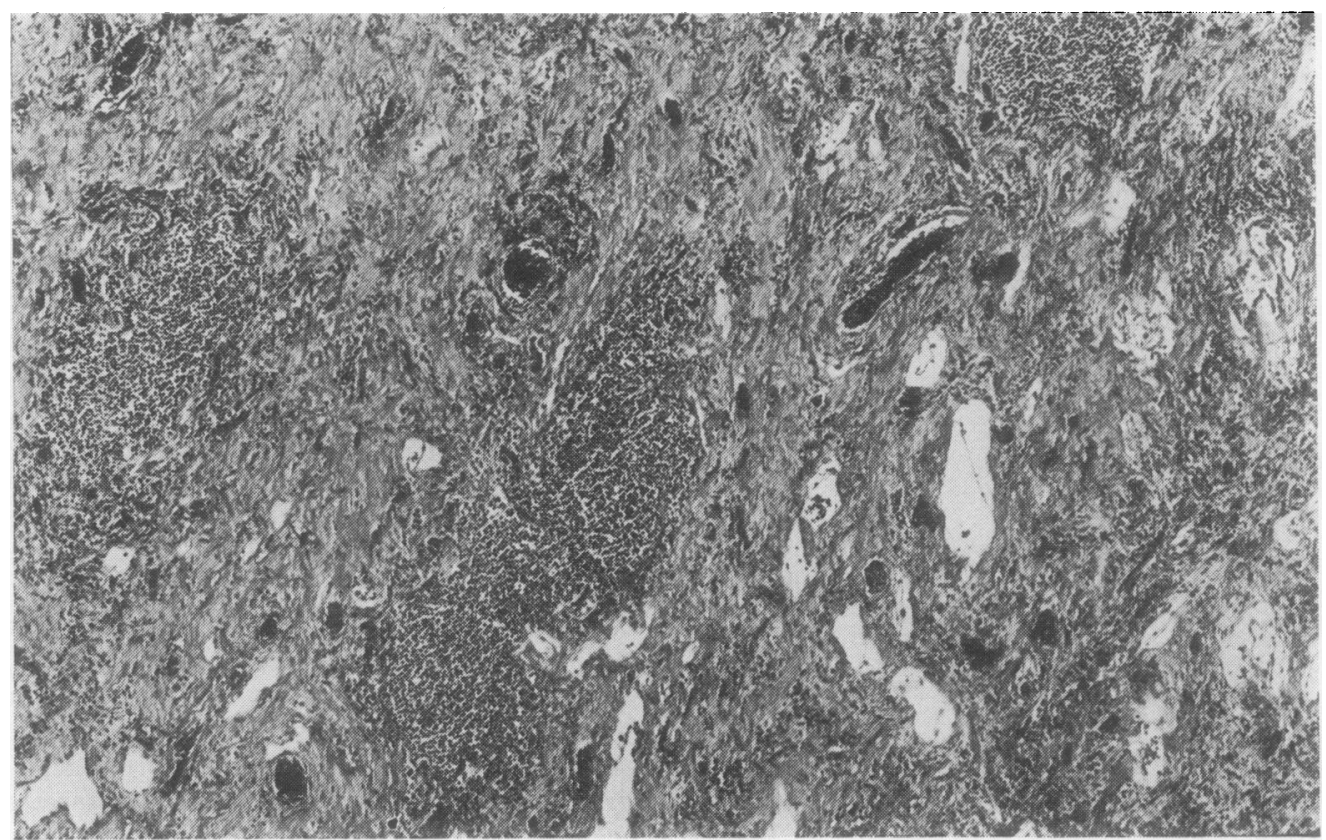

FIG. 4. Case 1. Right upper lobe: Dense hyaline fibrous tissue replacing lung architecture with only a few distorted air spaces remaining. Foci of lymphocytes at left margin, centrally and top right $(H$ and $E \times 44)$.

Over the years dyspnoea gradually increased but she is still able to cope with her housework. She has lost $6 \mathrm{~kg}$ in weight. Cough and sputum are mild and clubbing of the fingers has not appeared. The radiograph (Fig. 8) shows further deterioration with the development of bullae of variable size in both upper lobes, more fibrosis at the bases, and emphysema of the intervening areas.

She has not kept birds and has only taken simple medicaments for occasional dyspepsia.

In 1964 the VC and FEV 1 were 1.7 and 1.41 respectively. By 1973 these had fallen to 1.05 and 0.951.

Investigation 1964-69: Haemoglobin, white blood count, and platelets normal, ESR $60 \mathrm{~mm}$ and $45 \mathrm{~mm}$ in $1 \mathrm{hr}$; serum albumin $34 \mathrm{~g} / 1$, alpha globulin $4 \mathrm{~g} / 1$, alpha 2 globulin $10 \mathrm{~g} / 1$, beta globulin $11 \mathrm{~g} / 1$, and gamma globulin $17 \mathrm{~g} / 1$; Rose Waaler, latex, and antinuclear factor tests negative; $A$. fumigatus precipitin test negative; urea normal; tuberculin skin test negative; arterial $\mathrm{pH}$ and $\mathrm{PCO}_{2}$ normal; diffusing capacity (carbon monoxide, steady state) normal.

1970-73: ESR 23-54 $\mathrm{mm}$ in $1 \mathrm{hr}$; serum proteins and electrophoresis normal, alpha 1 antitryp- sin normal; liver function tests and serum calciump normal; precipitin tests with pigeon, budgerigar and hen sera negative; $A$. fumigatus prick tests. negative; Kveim test negative; electrocardiogram right atrial hypertrophy; no radiographic evidence of spondylitis.

CASE 4 J.S., born in 1928, worked as a clerk. His father and grandfather had bronchial asthma. Genitourinary system $\mathrm{He}$ began to get loin paing in 1948 and had some calcified opacities in thes right kidney and a normal intravenous pyelogram? Intermittent symptoms continued and in 1957 the pyelogram was again normal but acid-fast bacillp were grown from one specimen of urine. He was 0 treated with antituberculosis drugs for a year and no further evidence of tuberculosis was foundoSymptoms continued and in 1968 he had multiples calculi in the right kidney, and a calcium oxalate्ष stone obstructing the left ureter was removed $\stackrel{?}{?}$ Serum calcium, urea, and electrolytes were nor- 0 mal, as was the urinary calcium/creatinin ratio. He had no significant trouble with his urinary tract thereafter.

Respiratory system In 1952 he gave a history of some cough and purulent sputum since an illnesso 


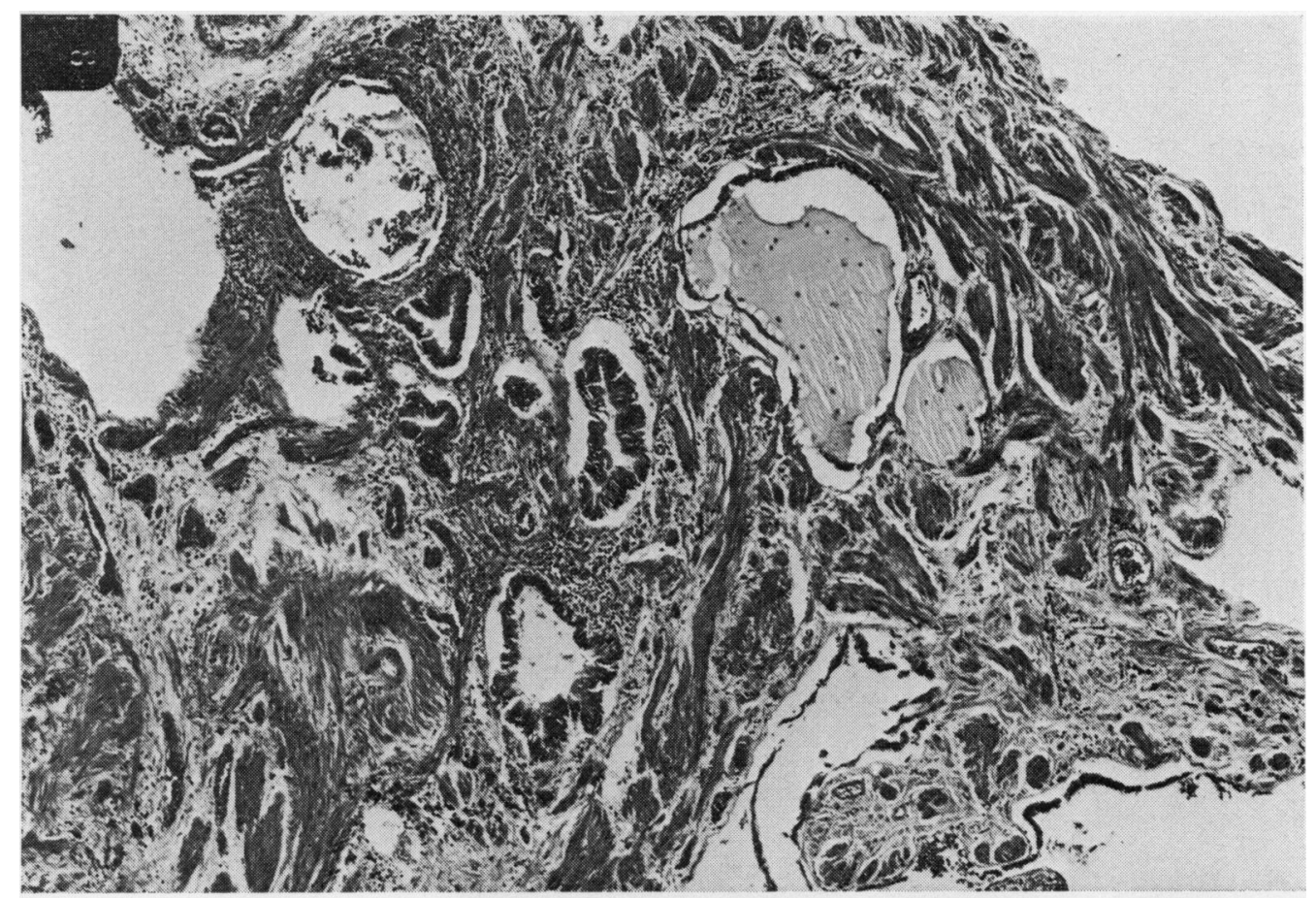

FIG. 5. Case 1. Right lower lobe. Muscular hyperplasia and honeycombing with epithelial metaplasia in air sacs, a picture resembling fibrosing alveolitis $(H$ and $E \times 44)$.

described as lung abscess two years previously. The only clinical abnormality was the presence of some wheezes over both lungs. Radiographs showed slight linear and nodular shadows in the upper third of the right lung and a small illdefined opacity above the left hilum. Bronchograms showed some bronchiectasis in the medial basal segment of the lower lobe and two small saccular areas of dilatation in the right upper lobe. On the left there were a few small scattered but well-localized cystic areas of dilatation.

Mild cough and sputum continued, and in 1957 he was treated for right-sided pneumonia. Examination afterwards showed bilateral wheezes and crackles, and radiographically there was an increase in linear shadow in both upper lobes. He gradually improved and returned to work. In 1961 he felt well with only a little cough and sputum. He smoked five cigarettes per day. Early next year his symptoms increased and the radiograph (Fig. 9) showed a considerable increase in linear shadowing in the upper two-thirds of both lungs with irregular cystic spaces, especially on the right. Thereafter he produced a lot of mucopurulent sputum and became steadily more short of breath and was able to work only intermittently. He came under our care in December 1969 when he was dyspnoeic and cyanosed at rest but had no finger clubbing. His sputum was grossly purulent. The radiograph showed extensive fibrosis and some consolidation throughout both lungs with many large cystic spaces, especially in the upper half of the right lung (Fig. 10). He failed to improve with antibiotics and died after nine days.

Investigations 1962: Haemoglobin and white blood count normal. ESR $16 \mathrm{~mm}$ in $1 \mathrm{hr}$. 196869: Mild polymorph leucocytosis with $13 \%$ and $11 \%$ eosinophils on two occasions; ESR $51 \mathrm{~mm}$ in $1 \mathrm{hr}$; WR negative; sputum cultures produced variable organisms including Haemophilus, Klebsiella, Entamoeba coli, and Candida albicans; no excess of eosinophils; aspergillus precipitin test negative; ECG, right ventricular hypertrophy; radiographs of pelvis, no evidence of ankylosing spondylitis. No history of avian contact. Tubercle bacilli were not found in 56 specimens of sputum.

December 1969: Arterial pH 7.36, $\mathrm{PCO}_{2}$ $7.05 \mathrm{kPa}(53 \mathrm{mmHg}), P_{2} 5.59 \mathrm{kPa}(42 \mathrm{mmHg})$, VC $1.21, \mathrm{FEV}_{1} 0.51$, PEFR $2051 / \mathrm{min}$. 
FIG. 6. Case 2. Radiograph in 1966 showing abnormal shadowing in periphery and apex of right upper lobe and in left upper/mid zone.
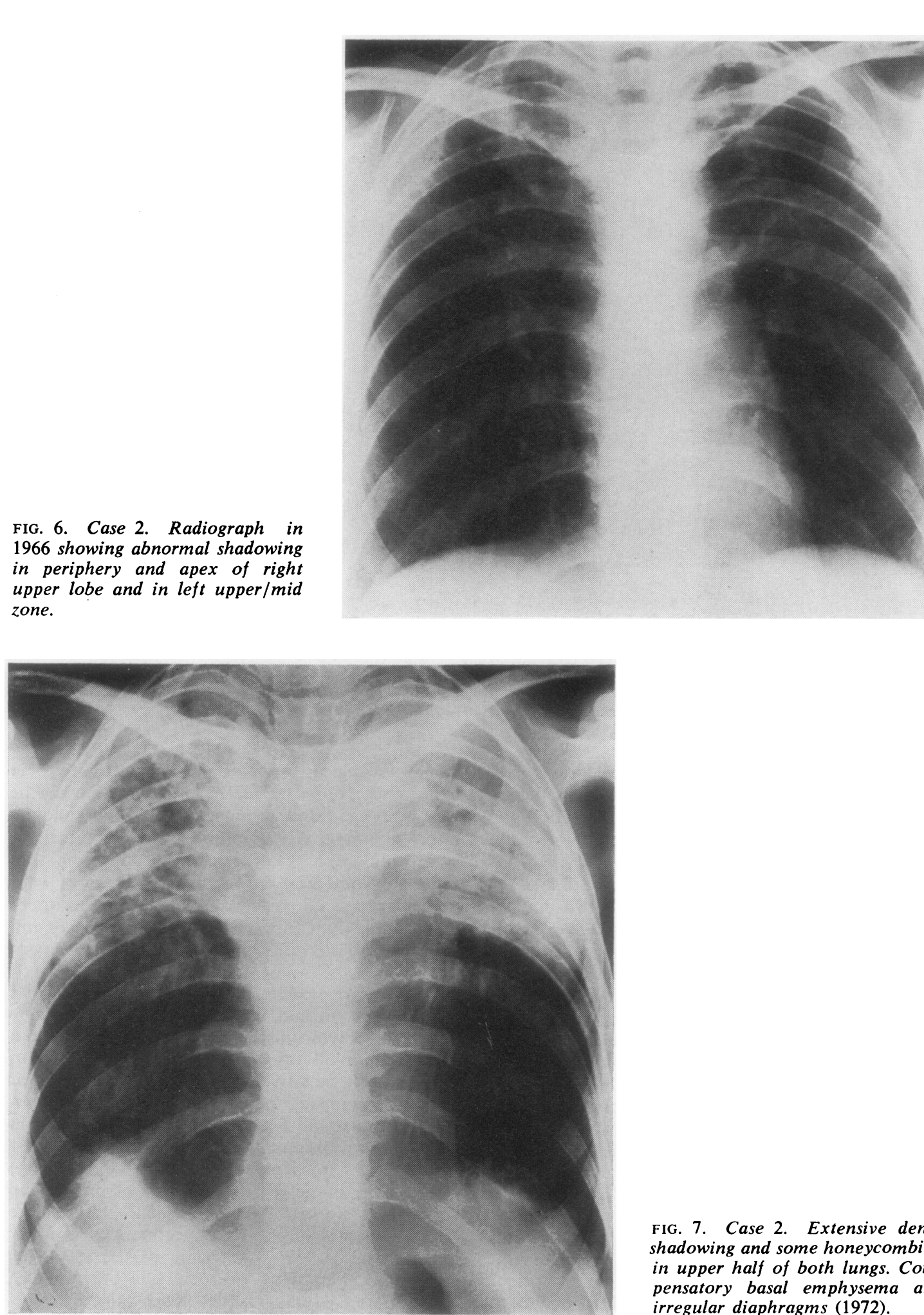

FIG. 7. Case 2. Extensive dens shadowing and some honeycombin in upper half of both lungs. Com? pensatory basal emphysema and irregular diaphragms (1972). 


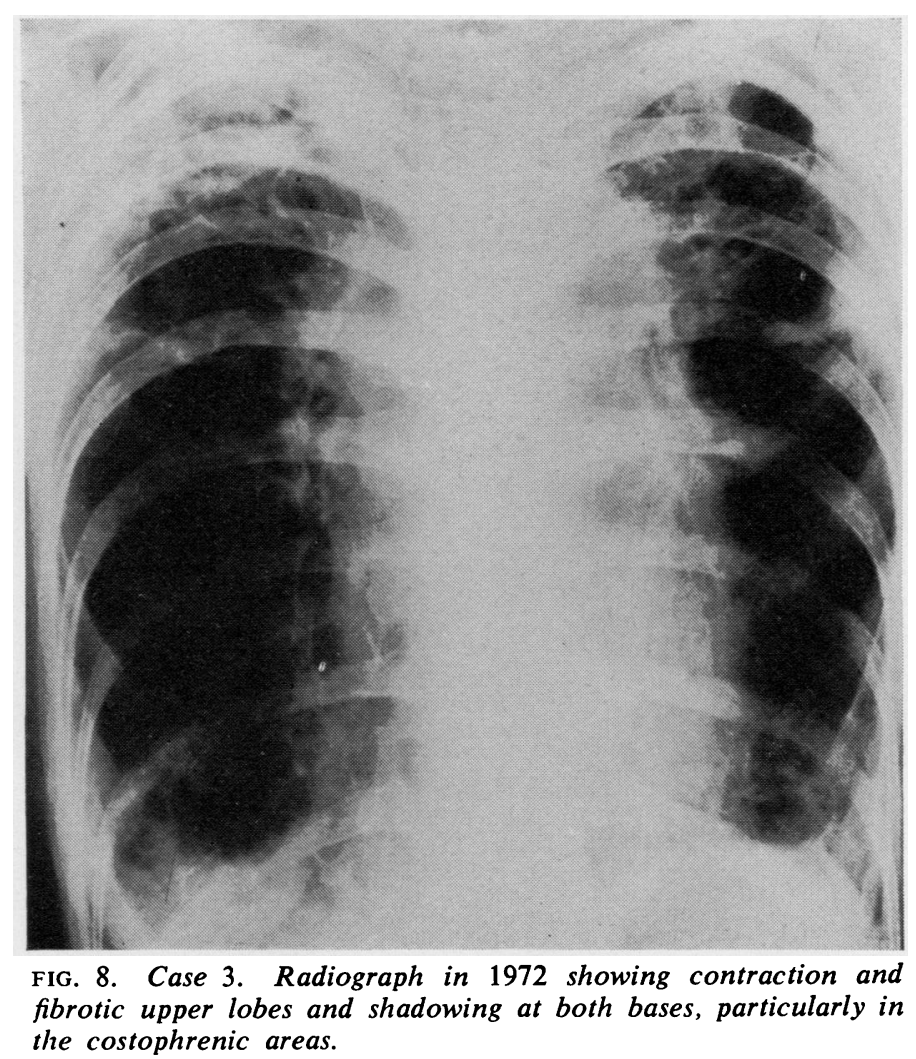

Necropsy The heart weighed $409 \mathrm{~g}$ and showed marked right ventricular hypertrophy. There was partial obliteration of the pleural cavities by fibrous adhesions. The right lung weighed $1100 \mathrm{~g}$ and the left $1170 \mathrm{~g}$. There were multiple irregular cavities on both sides with intervening fibrosis; one on the left contained pus. Some normal tissue remained in the lower lobes. No macroscopic abnormality was reported in the other organs.

Microscopically there was dense hyaline fibrosis in the affected areas with prominent lymphoid foci and severe endarteritis. The cavities were lined by low cubical epithelium. Elastic degeneration of collagen was prominent in some areas. An interstitial pattern of fibrosis was occasionally seen but the margins of the fibrotic areas were often clear cut.

CASE 5 A.M., born in 1911, worked as a maintenance engineer. He smoked 20 cigarettes per day. He presented in April 1972 with a six month's history of dyspnoea on exertion, a dry cough, and weight loss of $13 \mathrm{~kg}$. He was thin and had clubbing of the fingers. The trachea was displaced to the right and crackles were audible over both upper zones. The radiograph showed bilateral upper zone shadowing and cavitation on the right. The hila were displaced upwards, the costophrenic angles were obliterated, and the diaphragms were flat. Though tubercle bacilli were not found he was treated with streptomycin, PAS, and isoniazid for six months without radiographic improvement.

In March 1973 he was readmitted to hospital with increasing dyspnoea, more loss of weight, and melaena for two days. He had iron deficiency anaemia and a duodenal ulcer and he was given intravenous iron. There was also some extension of the shadowing on the left, and cavitation appeared in the upper zone. He was treated with rifampicin, ethambutol, and isoniazid. Though there was some improvement in his general condition the cavitation on the right increased.

Investigation Iron deficiency anaemia, with compatible bone marrow. Coombs' test negative. White blood count normal, ESR $30 \mathrm{~mm}$ in $1 \mathrm{hr}$; liver function tests normal; negative tests for 


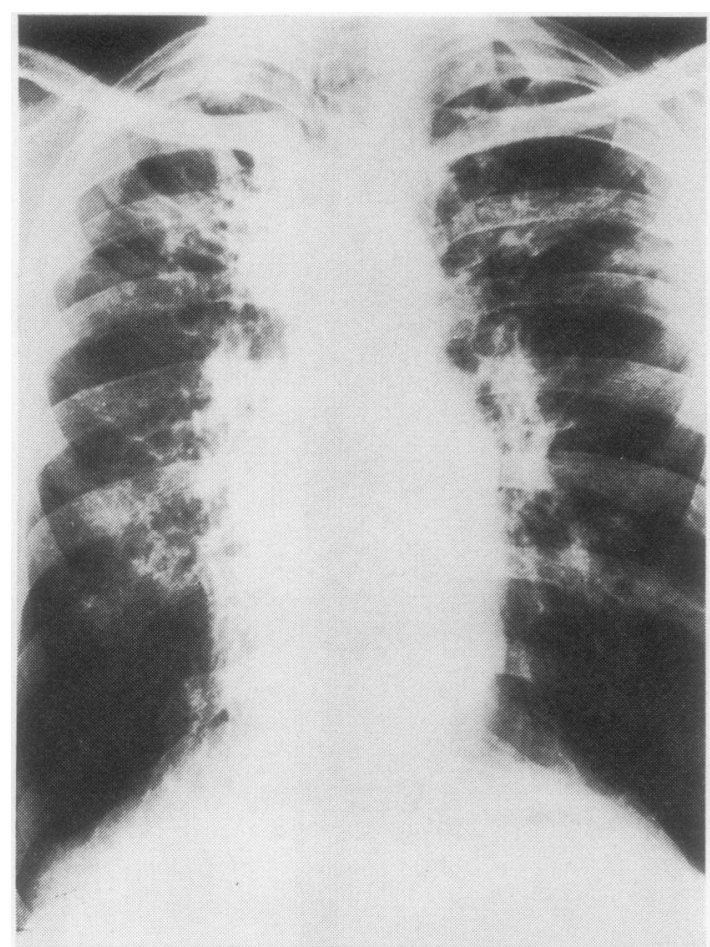

FIG. 9. Case 4. Radiograph in 1962 showing linear shadow in the upper two-thirds of both lungs and cystic spaces in the right lung.

rheumatoid and antinuclear factors, aspergillus, avian, and mouldy hay antibodies; tuberculin skin test positive, prick test with aspergillus and 15 other antigens negative; Kveim test negative; no radiographic evidence of ankylosing spondylitis; barium meal, duodenal ulcer. Repeated examinations of sputum for pyogenic organisms, mycobacteria, and fungi were negative.

\section{DISCUSSION}

The age at which lung changes were detected varied between 24 and 61 years, and the duration of the disease so far has ranged from two to 17 years, with two deaths. The early symptoms were cough and scanty sputum. Even after many years they were not dominant. The sputum remained mucoid with occasional episodes of purulence. The exception was case 4 whose early bronchograms showed localized areas of dilatation. The patient described in the Clinico-Pathological Conference (1962) also had recurrent infections but it was concluded that this was not the cause of the fibrosis.

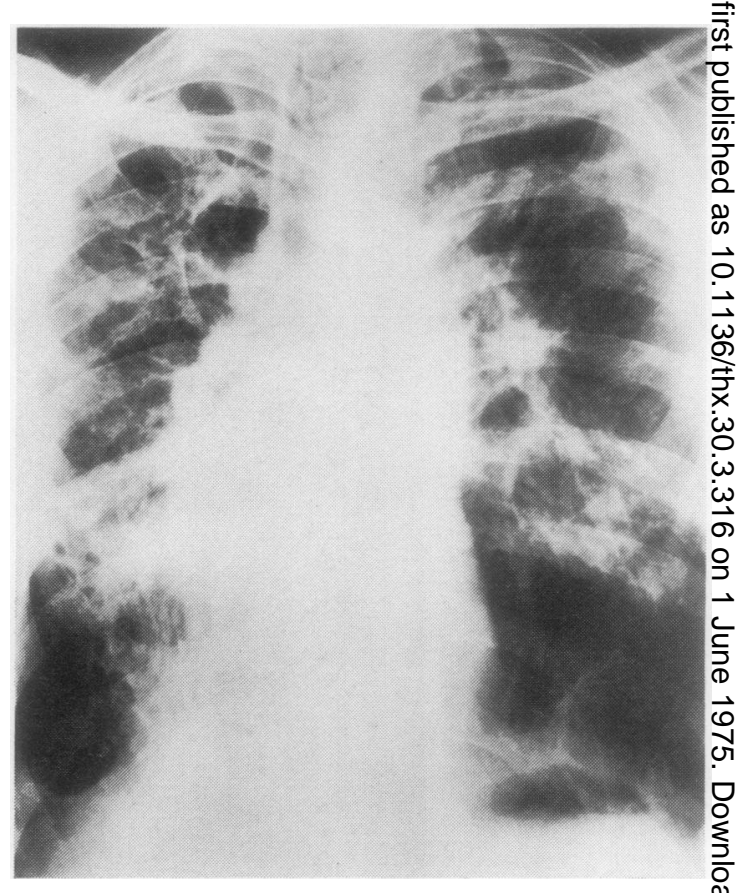

FIG. 10. Case 4. In 1969 there are large cystico spaces especially in the upper part of the right lung and scattered consolidation and fibrosis, most marked in the right mid zone.

Dyspnoea developed gradually. There was re strictive impairment of ventilation, except for some airways obstruction in case 4 and in the early stages of case 1 . The transfer factor was reduced in cases 1 and 2 . The unaffected parts of the lungs maintained normal or near-normal gas tensions and there was no severe ventilationperfusion inequality. However hypoxaemia and hypercapnia appeared in the terminal stages in cases 1 and 4 . Heart failure was not seen though there was evidence of right-sided hypertrophy Weight loss was a conspicuous feature. Finger clubbing was present in one case.

Radiographic abnormality was originally uni lateral in cases 1 and 2 but soon became bilateral $\omega$ The first changes were mainly apical. Progression occurred in all, mainly in the upper lobes. Ite case 1 there was a spread of softer shadowing tod the rest of the lungs after 10 years of progressive upper fibrosis. Case 3, however, developed some basal changes after three years. Case 4, startingt in the upper zones, developed extensive disease which still spared the bases.

The ESR and serum globulins were increased One patient had a weakly positive Rose Waale 
test and two had a temporary increase in blood eosinophils. There was no other evidence of autoallergy. Corticosteroids were given to three patients. One had symptomatic improvement but objective changes were not seen. Treatment was not started early enough nor continued long enough to see whether deterioration could be halted.

Other conditions besides tuberculosis may produce somewhat similar lung changes. Allergic aspergillosis (Henderson, 1968) was excluded, as was extrinsic allergic alveolitis due to vegetable dusts, bird droppings, and drugs. Negative Kveim tests and necropsies made sarcoidosis very unlikely.

Clinically and histologically the disease is somewhat similar to that found in ankylosing spondylitis, with some differences. Cavitation was less frequent and colonization with aspergilli was not seen, whereas these are common in spondylitis. The disease was also rather more widespread.

Histologically there was extensive dense nonspecific fibrosis with lymphoid collections. Bronchial dilatation seemed to be compensatory. In less affected areas there was interstitial fibrosis and other areas were normal. When cryptogenic fibrosing alveolitis affects a limited area this is usually in the lower lobes, whereas the upper zones were mainly involved here. In our experience, fibrosing alveolitis does not produce such confluent fibrosis and despite the basal findings in case 1 we reject this as a diagnosis. Case 1 was a coal miner but the fibrosis showed virtually no dust deposition and cannot be regarded as progressive massive fibrosis. The changes in the lower parts developed late and conformed histologically with fibrosing alveolitis. This was in clear contrast to the monotonous confluent fibrosis in the upper lobes. It is problematical as to whether this represented a second disease in the patient. Case 3 had widespread involvement at a fairly early stage but histology is not available. Neither she nor case 1 showed the dominant clinical features of fibrosing alveolitis with clubbing, widespread crepitations, and hypoxaemia.

Other diseases found were renal stones in one patient, a duodenal ulcer in another, and psoriasis in a third. The last was also present in a previously recorded case (Davies, 1970) but this may be a chance association.

The patients are grouped together because no cause was found for their disease and because they had many clinical and radiological features in common. The two patients who died also showed extensive unexplained collagenous fibrosis. However, we do not wish to imply that they all necessarily have a common pathogenesis. Our purpose is to draw attention to this unusual group and to encourage further study. Our experience and the previous report (Davies, 1970) suggest that this is not a very rare condition.

We are indebted to Dr. P. D. B. Davies for case 5 and to Mrs. B. Fancic for secretarial work.

\section{REFERENCES}

Clinico-Pathological Conference (1962). Undiagnosable lung disease. British Medical Journal, 1, 1403.

(1966). A case of idiopathic cavitation of lung. British Medical Journal, 1, 345.

Crompton, G. K., Cameron, S. J., and Langlands, A. O. (1974). Pulmonary fibrosis, pulmonary tuberculosis and ankylosing spondylitis. British Journal of Diseases of the Chest, 68, 51 .

Davies, D. (1970). Lung fibrosis and cavitation mimicking tuberculosis. Tubercle, 51, 246. (1972). Ankylosing spondylitis and lung fibrosis. Quarterly Journal of Medicine, 41, 395.

Henderson, A. H. (1968). Allergic aspergillosis. Review of thirty two cases. Thorax, 23, 501.

Requests for reprints to: Dr. D Davies, Ransom Hospital, Mansfield, Notts. 